\title{
Comparação internacional da eficiência ambiental dos modos de transporte rodoviário e ferroviário
}

[The environmental efficiency of road and rail freight modes in the world]

\author{
Pedrita Dantas Gabriele*, Luana Carneiro Brandão, Fernanda Tavares Treinta, \\ João Carlos C. B. Soares de Mello, Raquel Carvalhal \\ Universidade Federal Fluminense (UFF), Brazil
}

Submitted 26 Dec 2011; received in revised form 6 Feb 2012; accepted 10 Feb 2012

\begin{abstract}
Resumo
O despertar da sociedade para a sustentabilidade do meio ambiente impulsiona estudos que avaliem a eficiência ambiental das indústrias. Dentro deste contexto, se encontra o setor de transportes, que é um grande responsável pela emissão de gases provocadores de efeito estufa. Embora existam vários estudos relacionados à Análise Envoltória de Dados em Transportes e em Meio Ambiente, não foi observada na revisão da literatura uma aplicação desta metodologia especificamente para a análise da eficiência da emissão de $\mathrm{CO} 2$ na operação dos modos de transportes ferroviário e rodoviário no mundo. Por isso, o presente estudo apresenta uma modelagem DEA (Data Envelopment Analysis) para analisar a eficiência de 41 países da OCDE em relação à perspectiva ambiental de seus modos de transporte rodoviário e ferroviário. Vale ressaltar a relevância da utilização de modelos avançados em DEA no estudo, além da modelagem das emissões de CO2 como output indesejado. Os resultados indicam a eficiência de países pertencentes à União Europeia, com destaque para Letônia e Lituânia. Esses resultados foram confirmados por análises qualitativas, segundo a revisão da literatura.
\end{abstract}

Palavras-Chave: DEA; emissões de CO2; eficiência ambiental; transporte ferroviário; transporte rodoviário.

\begin{abstract}
The society awakening for the environment sustainability encourages studies that assess the industry environmental efficiency. Within this context, lies the transport sector, which consists of a large responsible for the emission of greenhouse gases provocateurs. Although several studies related to Data Envelopment Analysis in Transportation and Environment, was not observed in the literature review an application of this methodology specifically for the analysis of the CO2 emissions efficiency in the operation modes of rail and road transportation in the world. Therefore, this study presents a model DEA (Data Envelopment Analysis) to analyze the efficiency of 41 OECD countries in relation to the environmental perspective of their road and rail transport modes. It is worth emphasizing the importance of using advanced models in the DEA study, besides the modeling of $\mathrm{CO} 2$ emissions as unwanted output. The results indicate the efficiency of European Union countries, especially in Latvia and Lithuania. These results were confirmed by qualitative analysis, according to the literature review.
\end{abstract}

Key words: DEA; CO2 emissions; environmental efficiency; rail transportation; road transportation.

*Corresponding Author. Email: pedritadantas@gmail.com.

\section{Recommended Citation}

Gabriele, P. D., Brandão, L. C., Treinta, F. T., Mello, J. C. C. B. S., and Carvalhal, R. (2013) Comparação internacional da eficiência ambiental dos modos de transporte rodoviário e ferroviário. Journal of Transport Literature, vol. 7, n. 1, pp. 212-229.

- JTL/RELIT is a fully electronic, peer-reviewed, open access, international journal focused on emerging transport markets and published by BPTS - Brazilian Transport Planning Society. Website www.transport-literature.org. ISSN 2238-1031. 


\section{Introdução}

No contexto atual, a discussão relacionada às questões ambientais e ao desenvolvimento sustentável se encontra em posição de destaque, fazendo com que os diversos setores da sociedade, indústria e governo direcionassem esforços para tais assuntos. Sendo assim, ressalta-se a importância do setor de transportes na geração de impactos ambientais, principalmente devido às emissões gasosas geradoras do efeito estufa.

Nesse contexto, destaca-se ainda que desde a primeira Cúpula Mundial sobre Desenvolvimento Sustentável (CMDS), em 1992, o setor de transportes foi apontado como uma área chave, a fim de alcançar o desenvolvimento sustentável e reduzir os efeitos das mudanças climáticas.

Ao analisar o setor de transportes, nota-se que as questões relacionadas à globalização geraram um grande aumento do fluxo de conhecimentos, recursos, bens e serviços, o que levou a um aumento significativo ao longo dos anos em relação às atividades de transporte. (OECD, 2010).

Entretanto, ao mesmo tempo em que gera inúmeros benefícios para a sociedade, o setor de transportes também é responsável por grandes custos e impactos ambientais. Além disso, apesar do grande avanço no setor de transportes em relação ao desenvolvimento tecnológico nos últimos anos, nem sempre essas novas tecnologias estão alinhadas com os conceitos de desenvolvimento sustentável.

Dessa forma, tem-se que este setor apresenta um papel crucial dentro de uma economia global, impactando na competitividade da economia e na mobilidade de pessoas. Por outro lado, destaca-se importância de analisar o setor do ponto de vista das discussões ambientais. De acordo com a OECD (2008), tem-se que as emissões globais de $\mathrm{CO}_{2}$ do setor de transportes teve um crescimento de 44\% no período de 1990 até 2008. 
A partir desse contexto, o presente artigo tem como objetivo analisar a eficiência técnica em relação à perspectiva ambiental dos modos de transporte no mundo. Para este estudo, foi aplicado um modelo DEA com dados de transporte ferroviário e rodoviário de 41 países.

Ao analisar a questão ambiental e a preocupação com as emissões de gases, nota-se que as emissões de $\mathrm{CO}_{2}$ são vistas como um produto indesejado do processo produtivo, sendo assim, foram tratadas pela modelagem em DEA como outputs indesejáveis. Além disso, de acordo com as especificidades da modelagem, optou-se por utilizar o modelo híbrido não decrescente (Macedo, Mello e Gomes, 2010). Como resultado, houve destaque para países pertencentes à União Europeia, em especial a Lituânia e Letônia. Esses resultados foram confirmados por análises qualitativas, segundo a revisão da literatura. Portanto, este estudo encontra-se dividido em sete seções. Na seção 1 tem-se uma introdução que permite a contextualização em relação à temática do artigo. A seção 2 apresenta dados do setor de transportes no mundo. $\mathrm{Na}$ seção 3 tem-se uma revisão que relaciona o setor de transportes com as discussões ambientais. A seção 4 faz uma abordagem sobre os estudos de DEA, transporte e meio ambiente. Na seção 5 apresenta-se a modelagem DEA utilizada neste estudo e em seguida são apresentadas as principais análises dos resultados obtidos na seção 6. Por fim, a seção 7 aborda as principais conclusões relacionadas ao estudo.

\section{Transporte no mundo}

A escolha do modo de transporte de cargas e passageiros no país varia segundo aspectos políticos e históricos, como por exemplo, no Brasil, houve um incentivo a partir da década de 50 na construção de rodovias com o objetivo de estimular a entrada da indústria automotiva no país (Senge-sc, 2011). Ademais, existem influências comerciais e técnicas na escolha dos modos de transportes, como: o custo do transporte, o tempo médio de entrega, a variabilidade do tempo de entrega e as perdas e danos devido à insegurança no transporte. A Tabela 1 resume as médias de cada modo de transporte, segundo cada critério destacado. 
Tabela 1: Desempenho dos modos de transporte (Ballou, 2010)

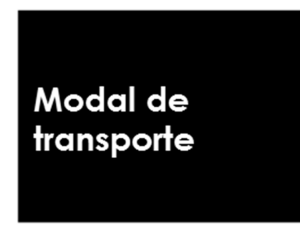

FERROVIÁRIO

RODOVIÁRIO

AQUAVIÁRIOS

DUTOVIÁRIO

AÉREO

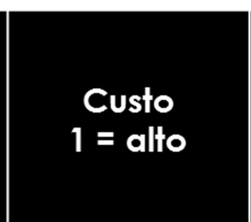

3

2

5

4

1

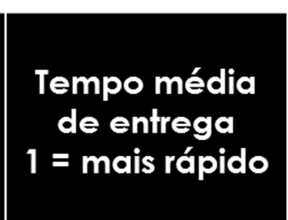

3

2

5

4

1

\section{Variabilidade do tempo de entrega (absoluto) em relação ao tempo médio de entrega 1 = menor}

\section{Perdas e}

danos

1 = menor

3

2

4

1

5
5

4

2

1

3

Dadas essas entre outras justificativas, o transporte de cargas e de passageiros no mundo, em geral, possui predominância conforme a região. Conforme observado na Tabela 2, exceto a Rússia, o transporte de passageiros por modo rodoviário prevalece em relação aos demais modos de transporte. Já o transporte de cargas, conforme observado na Tabela 3, é realizado, em sua maioria, por modo de transporte ferroviário e aquaviário.

Tabela 2: Distribuição do modo de transporte de passageiros no mundo (European Comission, 2010, GEIPOT, 2010 apud Andrade, Mattei, 2011)

\begin{tabular}{|c|c|c|c|c|c|}
\hline & Brasil (2005) & EUA (2007) & China (2007) & Rússia (2008) & União Européia (2008) \\
\hline Rodoviário & $96,18 \%$ & $87,8 \%$ & $54,1 \%$ & $26,2 \%$ & $82,7 \%$ \\
\hline Ferroviário & $1,37 \%$ & $0,7 \%$ & $33,3 \%$ & $47,8 \%$ & $7,8 \%$ \\
\hline Aquaviário & $0,00 \%$ & $0,0 \%$ & $0,3 \%$ & $0,2 \%$ & $0,6 \%$ \\
\hline Aeroviário & $2,45 \%$ & $11,5 \%$ & $12,3 \%$ & $25,8 \%$ & $8,8 \%$ \\
\hline
\end{tabular}

Tabela 3: Distribuição do modo de transporte de cargas no mundo (European Comission, 2010, CNT 2010 apud Andrade, Mattei, 2011)

\begin{tabular}{|l|c|c|c|c|c|}
\hline & Brasil (2006) & EUA (2007) & China (2007) & Rússia (2008) & União Européia (2008) \\
\cline { 2 - 5 } & $61,10 \%$ & $31,0 \%$ & $11,2 \%$ & $4,4 \%$ & $45,9 \%$ \\
\hline Rodoviário & $20,70 \%$ & $42,9 \%$ & $23,5 \%$ & $42,8 \%$ & $10,8 \%$ \\
\hline Ferroviário & $14,00 \%$ & $13,0 \%$ & $63,5 \%$ & $3,0 \%$ & $40,2 \%$ \\
\hline Dutoviáriário & $4,20 \%$ & $13,1 \%$ & $1,8 \%$ & $49,8 \%$ & $3,0 \%$ \\
\hline
\end{tabular}


Dada esta distribuição dos modos de transporte no mundo, o volume de emissões de gases de efeito estufa pelo setor de transporte, varia conforme o tipo e tecnologia envolvida, conforme apresentado no próximo capítulo.

\section{Transporte e meio ambiente}

De acordo com a OCDE (2008), o setor de transporte é o setor que mais emite gases de efeito estufa. O transporte rodoviário é a principal fonte desta emissão e os transportes marítimos e aéreos são os que menos contribuem com o total de emissões. Estatísticas do International Transport Forum indicam que o setor de transporte foi responsável por 22,5\% das emissões de $\mathrm{CO}_{2}$ do mundo em 2008 e que a emissão de $\mathrm{CO}_{2}$ aumentou $44 \%$ no período entre 1990 e 2008 .

Ainda, segundo a OCDE (2008), o nível de emissão de gases de efeitos estufa depende diretamente do tamanho, intensidade de uso e a tecnologia do veículo. Assim, países em que o uso é intensivo em transporte terrestre de carga e passageiros têm a propensão de poluírem mais quando se trata do setor de transporte. A Tabela 4 a seguir apresenta o percentual de utilização do meio de transporte por transporte de passageiros e cargas, por região no mundo.

Tabela 4: Modo de Transporte segregado por região, 2005.

\begin{tabular}{|c|c|c|c|c|c|c|c|c|c|}
\hline & \multicolumn{6}{|c|}{ Transporte de Passageiros } & \multicolumn{3}{|c|}{ Transporte de Cargas } \\
\hline Região & $\begin{array}{l}\text { Carro/ } \\
\text { Caminhão } \\
\text { Leve }\end{array}$ & Aéreo & Ferroviário & Ônibus & Outros & Total & Caminhão & Ferrovia & Total \\
\hline $\begin{array}{l}\text { OECD } \\
\text { América } \\
\text { do Norte }\end{array}$ & 81 & 14 & 1 & 4 & 0 & 100 & 40 & 60 & 100 \\
\hline $\begin{array}{l}\text { OECD } \\
\text { Europa }\end{array}$ & 63 & 16 & 5 & 13 & 3 & 100 & 86 & 14 & 100 \\
\hline $\begin{array}{l}\text { OECD } \\
\text { Pacífico }\end{array}$ & 56 & 13 & 9 & 16 & 7 & 100 & 72 & 28 & 100 \\
\hline China & 41 & 12 & 1 & 43 & 4 & 100 & 25 & 75 & 100 \\
\hline $\begin{array}{l}\text { América } \\
\text { Latina }\end{array}$ & 41 & 12 & 1 & 43 & 4 & 100 & 84 & 16 & 100 \\
\hline
\end{tabular}

Os dados informam que em todas as regiões, a utilização de ônibus e caminhões é maior do que os outros meios de transporte. E, o uso intensivo destes meios de transportes explica em grande parte a emissão de $\mathrm{CO}_{2}$ por países que compõem as regiões supracitadas. A Tabela 5 a seguir indica os maiores emissores de $\mathrm{CO}_{2}$ por transportes no mundo. 
Tabela 5: CO2 proveniente da queima de combustíveis no transporte

\begin{tabular}{llc}
\hline Ano & País & $\begin{array}{c}\text { CO2 da queima de } \\
\text { combustíveis (Mt) }\end{array}$ \\
\hline $\mathbf{2 0 0 8}$ & Estados Unidos & 1.834 \\
& China & 525 \\
& Russia & 260 \\
& Japão & 260 \\
& Alemanha & 179 \\
& Brasil & 168 \\
& Inglaterra & 167 \\
& Canada & 165 \\
& México & 164 \\
& França & 150 \\
\hline
\end{tabular}

Apesar do aumento da emissão de gases de efeitos estufa (GEE), o mundo está mais atento ao uso de tecnologias e recursos mais limpos para minimizar os efeitos da poluição. Bastos et al. (2007) sugere um sistema de comércio de emissão de gases poluentes combinado com a tributação das empresas devido às suas externalidades negativas para a redução das emissões de GEE. Enfim, no setor de transporte, existem inciativas e políticas importantes para alcançar um crescimento verde, que incluem o apoio de veículos elétricos, sistemas de renovação do parque automobilístico e investimentos em trens de alta velocidade já estão sendo implementadas em alguns países, entre outras ações (OCDE, 2008).

\section{DEA, Transporte e Meio Ambiente}

O presente capítulo busca apresentar a revisão da literatura realizada sobre a aplicação da modelagem DEA para a análise da eficiência dos modos de transporte e para análise da eficiência ambiental, especificamente em relação à emissão de gases de efeitos estufa. Entretanto, ressalta-se que este levantamento não pretende ser exaustivo.

Isso posto, segundo Tschaffon (2011) existem três formas de trabalhar com o output indesejável $\mathrm{CO}_{2}$ em DEA. Na primeira, utiliza-se o inverso do output indesejável como um output em DEA. Esta abordagem é chamada de inverso multiplicativo (Scheel, 2001, apud Tschaffon, 2011).

A segunda forma de aplicação foi desenvolvida por Golany e Roll (1989), em que utilizaram a translação de valores, adicionando à transformação no inverso aditivo um escalar positivo, 
grande o suficiente para que o valor final seja positivo. Esta abordagem somente é aplicável ao modelo BCC, já que o modelo CCR não é invariante a translação (Tschaffon, 2011).

Por último, Scheel (2001 apud Tschaffon, 2011) considera o output como um input (Reinhard et al., 2000 apud Tschaffon, 2011), que foi a abordagem utilizada no presente artigo.

Já Macedo, Mello e Gomes (2010) desenvolveram um modelo de redistribuição de cotas de emissão de gases do efeito estufa baseado em medidas de eficiência técnica, utilizando a ferramenta DEA com Ganhos de Soma Zero (DEA-GSZ). As variáveis de outputs foram número de habitantes, área $\left(106 \mathrm{~km}^{2}\right)$, energia consumida; e emissões de $\mathrm{CO}_{2}$ como output indesejável, que foi modelado como variável de input.

Ramanathan (2005) utilizou DEA para avaliar eficiência operacional e de emissão de gás de efeito estufa do transporte de passageiros e cargas nos modos rodoviário e ferroviário na Índia. Para isso, utilizou como variável de entrada a (1) energia utilizada e como variáveis de saída (1) passageiro*km e (2) toneladas*km.

Azevedo et al (2012) utilizaram DEA-BCC temporal para avaliar a eficiência das rodovias brasileiras no período de 2005 a 2008. Para isso, foram desenvolvidos dois modelos, em que o primeiro teve como inputs o número total de funcionários e o número total de veículos e como outputs o volume anual de carros e o número total de atendimentos. Já o segundo modelo teve como inputs acidentes $/ \mathrm{km}$ e receita anual $/ \mathrm{km}$ e como outputs as variáveis investimento acumulado/km e tráfego/km.

\section{Modelagem DEA}

A presente modelagem utilizou o output indesejável referente à $\mathrm{CO}_{2}$ como um input, medido em toneladas métricas. As demais variáveis referem-se ao produto do transporte rodoviário e ferroviário, de maneira semelhante à de Ramanathan (2005), isto é, o produto de passageiros rodoviários e ferroviários pela distância percorrida (em milhões de passageiro*km) - output 1 - e o produto da carga rodoviária e ferroviária pela distância percorrida (em milhões de toneladas*km) - output 2. As DMUs são os países da OCDE com dados disponíveis para 2008 e 1995. 
Para a avaliação da eficiência utilizou-se o SIAD (Angulo Meza et al, 2005) para rodar os dados de 2008, inicialmente. Em seguida, para analisar a evolução dessa eficiência, outro DEA foi rodado para 1995 e um terceiro para analisar conjuntamente 1995 e 2008. Ressaltase que não é possível avaliar comparativamente as DMUs em 1995 e em 2008 em modelos separados, uma vez que uma maior média de eficiência em um modelo não significa melhores DMUs, mas apenas DMUs mais homogêneas (Gomes et al, 2009).

Para esses modelos, foi utilizado inicialmente o BCC, uma vez que as DMUs apresentam tamanhos bastante diferentes entre si e que não há linearidade comprovada entre os inputs e os outputs. O CCR foi utilizado apenas para relativizar a benevolência do BCC, com o qual se conclui que este último, no presente caso, pode beneficiar DMUs de grande porte. Dessa maneira, utilizou-se por fim o modelo híbrido não decrescente (Macedo, Mello e Gomes, 2010).

Utiliza-se a orientação a input, uma vez que o objetivo é reduzir as emissões de $\mathrm{CO}_{2}$, dadas as demandas de transporte de passageiros e de carga para o país. Ressalta-se que modelos avançados em DEA como a fronteira invertida, a avaliação cruzada e o MCDEA (Li e Reeves, 1999) não devem ser usados porque os mesmos prejudicam DMUs boas em certos critérios e ruins em outros (DMUs específicas) e beneficiam aquelas mais homogêneas. Neste caso, deve haver preferência de países com demandas semelhantes entre carga e passageiros, em detrimento daqueles mais voltados para um tipo de transporte.

\section{Análise de resultados}

O resultado com o BCC e o CCR para as DMUs com dados de 2008 estão representados na Tabela 6. 
Tabela 6: Resultados do DEA BCC e do DEA CCR para os países da OCDE com dados de 2008

\begin{tabular}{|c|c|c|c|c|c|}
\hline 2008 & Input1 & Output1 & Output2 & $\overline{\mathrm{BCC}}$ & $\overline{C C R}$ \\
\hline Albania & 4.24 & 6488 & 4150 & 0,872068 & 0,545954 \\
\hline Armenia & 5.43 & 2548 & 1805 & 0,568223 & 0,167421 \\
\hline Australia & 409.64 & 284637 & 388346 & 0,78604 & 0,341694 \\
\hline Azerbaijan & 30.59 & 15088 & 20338 & 0,265989 & 0,240425 \\
\hline Belarus & 64.19 & 16408 & 71761 & 0,582155 & 0,327793 \\
\hline Belgium & 147.5 & 141673 & 46825 & 0,416156 & 0,342694 \\
\hline Bosnia and Herzegovina & 19.55 & 2191 & 3115 & 0,170648 & 0,056801 \\
\hline Bulgaria & 49.79 & 13733 & 11815 & 0,125311 & 0,09911 \\
\hline Canada & 554.24 & 497527 & 426000 & 0,741846 & 0,321596 \\
\hline Croatia & 21.15 & 5903 & 14354 & 0,267762 & 0,215513 \\
\hline Czech Republic & 117.82 & 88508 & 66314 & 0,421014 & 0,268025 \\
\hline Denmark & 54.06 & 74782 & 12581 & 0,560358 & 0,493551 \\
\hline Estonia & 18.49 & 2950 & 14222 & 0,295397 & 0,223353 \\
\hline Finland & 59.56 & 74992 & 38391 & 0,510251 & 0,449233 \\
\hline France & 393.85 & 856983 & 236031 & 1 & 0,776341 \\
\hline Germany & 834.95 & 992818 & 457202 & 0,769158 & 0,424248 \\
\hline Greece & 106.05 & 44185 & 17746 & 0,15155 & 0,148654 \\
\hline Hungary & 53.83 & 65317 & 22884 & 0,481028 & 0,432925 \\
\hline Iceland & 2.74 & 5585 & 0 & 1 & 0,72725 \\
\hline Italia & 447.84 & 891890 & 213610 & 0,998317 & 0,710558 \\
\hline Japan & 1185.66 & 1161772 & 368676 & 0,724807 & 0,3496 \\
\hline Korea & 541.71 & 236364 & 116788 & 0,235616 & 0,155677 \\
\hline Latvia & 8.85 & 3468 & 31925 & 1 & 1 \\
\hline Lithuania & 14.75 & 41341 & 35167 & 1 & 1 \\
\hline Mexico & 420.9 & 464043 & 301872 & 0,757604 & 0,39336 \\
\hline Netherlands & 237.46 & 178549 & 41328 & 0,330677 & 0,268274 \\
\hline Norway & 40.23 & 61355 & 18630 & 0,597869 & 0,54414 \\
\hline Poland & 301.15 & 318252 & 226266 & 0,746563 & 0,37705 \\
\hline Portugal & 57.44 & 100570 & 19317 & 0,736053 & 0,624691 \\
\hline Republic of Moldova & 7.11 & 3084 & 5839 & 0,542546 & 0,273583 \\
\hline Romania & 90.51 & 20761 & 38419 & 0,165451 & 0,142097 \\
\hline Russian Federation & 1611.17 & 291236 & 2332516 & 1 & 0,404456 \\
\hline Serbia & 49.35 & 5468 & 5451 & 0,077348 & 0,043789 \\
\hline Slovak Republic & 36.42 & 35241 & 38393 & 0,449332 & 0,405944 \\
\hline Slovenia & 17.04 & 28858 & 6155 & 0,619549 & 0,604238 \\
\hline Spain & 355.43 & 426819 & 248943 & 0,766959 & 0,428451 \\
\hline Sweden & 54.62 & 114056 & 53908 & 0,898921 & 0,745036 \\
\hline Switzerland & 47.87 & 104350 & 28483 & 0,919903 & 0,77775 \\
\hline Ukraine & 310.36 & 108502 & 275175 & 0,624818 & 0,279546 \\
\hline United Kingdom & 553.25 & 779928 & 187283 & 0,64715 & 0,502972 \\
\hline United States & 5738.37 & 4361392 & 4517589 & 1 & 0,308147 \\
\hline
\end{tabular}

Embora a modelagem sugira retornos decrescentes de escala, o que orienta a modelagem BCC, a análise da Tabela 16 leva à conclusão de que o BCC pode estar beneficiando países pequenos como a Letônia, a Lituânia e a Islândia, bem como países grandes como a Rússia e os EUA. Os resultados do CCR, com apenas a Letônia e a Lituânia eficientes descarta a possibilidade de o BCC beneficiar as pequenas DMUs. Entretanto, como as eficiências da 
Rússia e dos EUA caíram para $40 \%$ e $31 \%$, respectivamente, a possibilidade de o BCC beneficiar grandes DMUs fica mantida. O CCR assim sugere que pequenos países utilizam seus recursos ambientais de forma mais eficiente. Conforme já mencionado, essa situação sugere o uso do modelo híbrido, cujos resultados serão analisados posteriormente.

Outra característica do modelo importante é a queda brusca das eficiências ao longo do ranking no CCR, uma vez que há duas DMUs eficientes e a terceira melhor DMU apresenta eficiência de 77,7\% (Suíça). Essa situação sugere o uso do DEA em camadas porque as duas DMUs eficientes podem estar em um nível muito superior de tecnologia e prejudicando a liberdade em DEA das demais e conseqüentemente a sua ordenação (Barr et al, 2000).

Assim, a Tabela 7 apresenta os resultados para a $2^{\text {a }}$ Camada do DEA CCR, isto é, para o novo DEA CCR rodado sem as DMUs eficientes do modelo anterior - Letônia e Lituânia. Observase que a Rússia passou a ser eficiente e as demais DMUs alteraram bastante a sua ordem interna. Os EUA passaram a ter eficiência de $65 \%$, em outras palavras, ainda permanecem como ineficientes de escala - apresentam a gestão eficiente, com base no resultado do BCC, mas seu tamanho não é propício para um bom resultado geral, com base no resultado do CCR (Barr et al, 2000).

As eficiências do ranking estão bem mais homogêneas - a média do CCR com as 41 DMUs iniciais é de $41 \%$ e com as 39 DMUs da segunda camada é de 61,1\%. Como o objetivo da aplicação do DEA em camadas era apenas para complementar as análises iniciais, não será dada seqüência para o processo do DEA em camadas. 
Tabela 7: Resultados do DEA CCR para a $2^{\mathrm{a}}$ Camada

\begin{tabular}{|lc|}
\hline 2a Camada & CCR \\
Russian Federation & 1 \\
Sweden & 1 \\
Switzerland & 1 \\
France & 0,999 \\
Iceland & 0,935 \\
Italia & 0,914 \\
Albania & 0,904 \\
Slovak Republic & 0,864 \\
Portugal & 0,803 \\
Belarus & 0,791 \\
Slovenia & 0,777 \\
Australia & 0,748 \\
Norway & 0,704 \\
Poland & 0,675 \\
Spain & 0,66413 \\
Canada & 0,66077 \\
Mexico & 0,65947 \\
Ukraine & 0,65113 \\
United States & 0,65098 \\
United Kingdom & 0,6467 \\
Finland & 0,63614 \\
Denmark & 0,63459 \\
Republic of Moldova & 0,62091 \\
Germany & 0,56798 \\
Hungary & 0,56611 \\
Estonia & 0,54159 \\
Azerbaijan & 0,52569 \\
Croatia & 0,50027 \\
Czech Republic & 0,49907 \\
Japan & 0,45389 \\
Belgium & 0,4462 \\
Netherlands & 0,34494 \\
Romania & 0,32177 \\
Armenia & 0,29889 \\
Korea & 0,21523 \\
Bulgaria & 0,20379 \\
Greece & 0,19654 \\
Bosnia and Herzegov & 0,12499 \\
Serbia & 0,09201 \\
\hline
\end{tabular}

Os benchmarks do DEA BCC com as 41 DMUs estão representados na Tabela 8. Os EUA são benchmark de muito poucas DMUs, dado o seu gigantismo, não compartilhado pelos demais.

Ressalta-se que não cabe a avaliação de benchmarks com base no CCR porque as únicas DMUs eficientes e que são, portanto, as únicas possibilidades de benchmark são Letônia e Lituânia, bastante diferentes dos demais países. O resultado não fará muito sentido prático. 
Tabela 8: Benchmarks, com base no BCC.

\begin{tabular}{|c|c|c|c|c|c|c|}
\hline Benchmarks & France & Iceland & Latvia & Lithuania & Russian Federation & United States \\
\hline Albania & 0 & 0,873149 & 0,095917 & 0,030933 & 0 & 0 \\
\hline Armenia & 0 & 0,943461 & 0,056539 & 0 & 0 & 0 \\
\hline Australia & 0,258101 & 0 & 0 & 0,610732 & 0,13116674 & 0 \\
\hline Azerbaijan & 0 & 0,391877 & 0,323213 & 0,28491 & 0 & 0 \\
\hline Belarus & 0 & 0 & 0,770609 & 0,212375 & 0,01701627 & 0 \\
\hline Belgium & 0,12301 & 0 & 0 & 0,87699 & 0 & 0 \\
\hline Bosnia and Herzegovina & 0 & 0,902428 & 0,097572 & 0 & 0 & 0 \\
\hline Bulgaria & 0 & 0,653727 & 0,111777 & 0,234496 & 0 & 0 \\
\hline Canada & 0,521135 & 0 & 0 & 0,354306 & 0,12455914 & 0 \\
\hline Croatia & 0 & 0,553769 & 0,412891 & 0,03334 & 0 & 0 \\
\hline Czech Republic & 0,055152 & 0 & 0 & 0,936113 & 0,00873573 & 0 \\
\hline Denmark & 0,041 & 0 & 0 & 0,959 & 0 & 0 \\
\hline Estonia & 0 & 0,554518 & 0,445482 & 0 & 0 & 0 \\
\hline Finland & 0,041257 & 0 & 0 & 0,958743 & 0 & 0 \\
\hline France & 1 & 0 & 0 & 0 & 0 & 0 \\
\hline Germany & 0,938235 & 0 & 0 & 0 & 0,0198059 & 0,04195861 \\
\hline Greece & 0,003487 & 0 & 0 & 0,996513 & 0 & 0 \\
\hline Hungary & 0,029395 & 0 & 0 & 0,970605 & 0 & 0 \\
\hline Iceland & 0 & 1 & 0 & 0 & 0 & 0 \\
\hline Italia & 0,990039 & 0 & 0 & 0 & 0 & 0,00996088 \\
\hline Japan & 0 & 0 & 0 & 0 & 0 & 0,07778696 \\
\hline Korea & 0,2345 & 0 & 0 & 0,750474 & 0,01502529 & 0 \\
\hline Latvia & 0 & 0 & 1 & 0 & 0 & 0 \\
\hline Lithuania & 0 & 0 & 0 & 1 & 0 & 0 \\
\hline Mexico & 0,495962 & 0 & 0 & 0,431309 & 0,07272909 & 0 \\
\hline Netherlands & 0,168221 & 0 & 0 & 0,831779 & 0 & 0 \\
\hline Norway & 0,024538 & 0 & 0 & 0,975462 & 0 & 0 \\
\hline Poland & 0,322659 & 0 & 0 & 0,62237 & 0,0549714 & 0 \\
\hline Portugal & 0,072616 & 0 & 0 & 0,927384 & 0 & 0 \\
\hline Republic of Moldova & 0 & 0,817103 & 0,182897 & 0 & 0 & 0 \\
\hline Romania & 0 & 0 & 0,55793 & 0,439867 & 0,00220289 & 0 \\
\hline Russian Federation & 0 & 0 & 0 & 0 & 1 & 0 \\
\hline Serbia & 0 & 0,829908 & 0,163674 & 0,006418 & 0 & 0 \\
\hline Slovak Republic & 0 & 0 & 0,171931 & 0,826422 & 0,00164685 & 0 \\
\hline Slovenia & 0 & 0,349116 & 0 & 0,650884 & 0 & 0 \\
\hline Spain & 0,456321 & 0 & 0 & 0,490523 & 0,05315584 & 0 \\
\hline Sweden & 0,089036 & 0 & 0 & 0,910591 & 0,00037295 & 0 \\
\hline Switzerland & 0,077251 & 0 & 0 & 0,922749 & 0 & 0 \\
\hline Ukraine & 0,051719 & 0 & 0 & 0,848331 & 0,09994979 & 0 \\
\hline United Kingdom & 0,905528 & 0 & 0 & 0,094472 & 0 & 0 \\
\hline United States & 0 & 0 & 0 & 0 & 0 & 1 \\
\hline
\end{tabular}

Para realizar a avaliação temporal, rodou-se primeiramente DEA BCC para as 37 DMUs com dados disponíveis para 1990, cujos resultados estão apresentados na Tabela 9. Em seguida, rodou-se novamente DEA BCC para essas mesmas 37 DMUs, mas com os dados de 2008, para fins comparativos. Por fim, rodou-se o DEA BCC para as 74 DMUs conjuntamente - 37 com dados de 1990 e as outras 37 com dados 2008. 
Tabela9: DEA BCC para 1990

\begin{tabular}{|l|l|r|r|r|r|}
\hline & Ingut1 & Output1 & Output2 & BCC & CCR \\
\hline Albania & 6.25 & 2953 & 1779 & 0,563692 & 0,337276 \\
\hline Australia & 266.51 & 218980 & 169550 & 0,754528 & 0,685254 \\
\hline Azerbaijan & 63.91 & 7480 & 40363 & 0,513937 & 0,494775 \\
\hline Belarus & 123.97 & 36639 & 97791 & 0,655753 & 0,645193 \\
\hline Belgium & 123.68 & 98697 & 40403 & 0,470186 & 0,463759 \\
\hline Bosnia and Herzegovina & 23.71 & 4119 & 7075 & 0,314711 & 0,259477 \\
\hline Bulgaria & 75.83 & 38223 & 27902 & 0,408414 & 0,404772 \\
\hline Croatia & 21.87 & 10433 & 9387 & 0,466225 & 0,439703 \\
\hline Denmark & 6.25 & 7029 & 1779 & 0,705994 & 0,563848 \\
\hline Estonia & 266.51 & 220490 & 169550 & 0,756991 & 0,686912 \\
\hline Finland & 57.29 & 71053 & 21697 & 0,653565 & 0,652683 \\
\hline France & 63.91 & 71241 & 40363 & 0,780903 & 0,767768 \\
\hline Germany & 123.97 & 19787 & 97791 & 0,627656 & 0,619804 \\
\hline Greece & 80.44 & 26210 & 13095 & 0,210054 & 0,209168 \\
\hline Hungary & 67.23 & 82402 & 31940 & 0,701485 & 0,696977 \\
\hline Iceland & 2.2 & 3004 & 0 & 1 & 0,664605 \\
\hline Italia & 410.23 & 652061 & 199115 & 1 & 0,836487 \\
\hline Japan & 1095.34 & 1090611 & 301440 & 0,9459 & 0,510246 \\
\hline Korea & 235.41 & 29863 & 13663 & 0,078164 & 0,077701 \\
\hline Latvia & 20.34 & 11228 & 24391 & 1 & 1 \\
\hline Lithuania & 33.81 & 10317 & 26594 & 0,652615 & 0,645299 \\
\hline Mexico & 272.12 & 276848 & 145301 & 0,768536 & 0,671043 \\
\hline Netherlands & 194.43 & 161460 & 25961 & 0,464964 & 0,404192 \\
\hline Norway & 30.92 & 47327 & 9864 & 0,756695 & 0,744999 \\
\hline Poland & 345.74 & 164773 & 123823 & 0,420124 & 0,389867 \\
\hline Portugal & 42.68 & 56464 & 12510 & 0,652242 & 0,643923 \\
\hline Republic of Moldova & 30.4 & 4878 & 21088 & 0,588273 & 0,548801 \\
\hline Romania & 167.77 & 54589 & 62461 & 0,36351 & 0,355528 \\
\hline Russian Federation & 2211.02 & 536152 & 2822277 & 1 & 1 \\
\hline Serbia & 61.87 & 34589 & 15789 & 0,344718 & 0,342022 \\
\hline Slovenia & 12.58 & 21257 & 9096 & 1 & 1 \\
\hline Spain & 220.59 & 223241 & 102143 & 0,704348 & 0,619891 \\
\hline Sweden & 55.91 & 101676 & 36091 & 1 & 1 \\
\hline Switzerland & 43.77 & 89927 & 19998 & 1 & 1 \\
\hline Ukraine & 693.97 & 166361 & 488747 & 0,58255 & 0,571528 \\
\hline United Kingdom & 575.95 & 666781 & 148900 & 0,74874 & 0,564002 \\
\hline United States & 4998.13 & 3866914 & 2748759 & 1 & 0,610921 \\
\hline
\end{tabular}

As 37 DMUs de 1990 são mais homogêneas que as 37 DMUs de 2008, uma vez que a média de eficiência para 1990 é de 59,8\%, enquanto que para 2008 essa média é de 42,6\%. Entretanto, isso não significa que as primeiras são melhores, o que pode ser definido apenas pela avaliação conjunta com as 74 DMUs. Com base nessa última análise, a média das DMUs de 1990 é de 32,6\%, enquanto a média de 2008 é de 42,6\%. Além disso, todas as DMUs eficientes no modelo de 2008 também foram eficientes no modelo conjunto, enquanto apenas 
2 das 7 DMUs eficientes no modelo de 1990 foram eficientes no modelo conjunto. Essa situação permite concluir que as DMUs de 2008 são melhores, apesar de menos homogêneas.

O resultado do modelo híbrido não decrescente para as 41 DMUs originais está representado na Tabela 10.

Tabela 10: Resultados do DEA BCC, CCR e Híbrido não decrescente.

\begin{tabular}{|c|c|c|c|}
\hline DMU & BCC & CCR & Híbrido \\
\hline Albania & 0,872068 & 0,545954 & 0.8720675 \\
\hline Armenia & 0,568223 & 0,167421 & 0.5682232 \\
\hline Australia & 0,78604 & 0,341694 & 0.3416937 \\
\hline Azerbaijan & 0,265989 & 0,240425 & 0.2659889 \\
\hline Belarus & 0,582155 & 0,327793 & 0.3277930 \\
\hline Belgium & 0,416156 & 0,342694 & 0.3426937 \\
\hline Bosnia and Herzegovina & 0,170648 & 0,056801 & 0.1706480 \\
\hline Bulgaria & 0,125311 & 0,09911 & 0.1253114 \\
\hline Canada & 0,741846 & 0,321596 & 0.3215956 \\
\hline Croatia & 0,267762 & 0,215513 & 0.2677623 \\
\hline Czech Republic & 0,421014 & 0,268025 & 0.2680245 \\
\hline Denmark & 0,560358 & 0,493551 & 0.4935510 \\
\hline Estonia & 0,295397 & 0,223353 & 0.2953971 \\
\hline Finland & 0,510251 & 0,449233 & 0.4492326 \\
\hline France & 1 & 0,776341 & 0.7763408 \\
\hline Germany & 0,769158 & 0,424248 & 0.4242484 \\
\hline Greece & 0,15155 & 0,148654 & 0.1486535 \\
\hline Hungary & 0,481028 & 0,432925 & 0.4329252 \\
\hline Iceland & 1 & 0,72725 & 1 \\
\hline Italia & 0,998317 & 0,710558 & 0.7105579 \\
\hline Japan & 0,724807 & 0,3496 & 0.3496003 \\
\hline Korea & 0,235616 & 0,155677 & 0.1556774 \\
\hline Latvia & 1 & 1 & 1 \\
\hline Lithuania & 1 & 1 & 1 \\
\hline Mexico & 0,757604 & 0,39336 & 0.3933601 \\
\hline Netherlands & 0,330677 & 0,268274 & 0.2682737 \\
\hline Norway & 0,597869 & 0,54414 & 0.5441404 \\
\hline Poland & 0,746563 & 0,37705 & 0.3770503 \\
\hline Portugal & 0,736053 & 0,624691 & 0.6246907 \\
\hline Republic of Moldova & 0,542546 & 0,273583 & 0.5425462 \\
\hline Romania & 0,165451 & 0,142097 & 0.1420973 \\
\hline Russian Federation & 1 & 0,404456 & 0.4044563 \\
\hline Serbia & 0,077348 & 0,043789 & 0,077348 \\
\hline Slovak Republic & 0,449332 & 0,405944 & 0.4059441 \\
\hline Slovenia & 0,619549 & 0,604238 & 0.6195490 \\
\hline Spain & 0,766959 & 0,428451 & 0.4284506 \\
\hline Sweden & 0,898921 & 0,745036 & 0.7450364 \\
\hline Switzerland & 0,919903 & 0,77775 & 0.7777501 \\
\hline Ukraine & 0,624818 & 0,279546 & 0.2795465 \\
\hline United Kingdom & 0,64715 & 0,502972 & 0.6383593 \\
\hline United States & 1 & 0,308147 & 0.3081472 \\
\hline
\end{tabular}

O modelo híbrido resultou em apenas uma DMU eficiente a mais que o CCR (Islândia) e apenas 12 das 41 DMUs estão na região de retornos crescentes de escala, isto é, mantiveram 
os valores do BCC. Além disso, a média de eficiência do modelo híbrido (45,6\%) aproximase mais do modelo CCR $(41,7 \%)$ que do modelo BCC $(60,5 \%)$.

Ressalta-se que o objetivo dessas análises complementares - modelo híbrido e DEA em Camadas - não apresentam como objetivo fornecer um ranking mais adequado que o DEA BCC original, mas apenas oferecer insumos para uma melhor análise da situação real desses países.

Por fim, ressalta-se que em todas as aplicações das modelagens DEA houve destaque para os países Letônia e Lituânia. Segundo ECORYS Transport (2006a), embora o transporte de passageiros na Letônia seja realizado pelo modo rodoviário, este país consiste em um dos mais pobres da Europa, tendo como consequência uma baixa utilização do transporte de passageiros. Além disso, o transporte de cargas consiste em um setor-chave da economia do país, que possui uma grande malha ferroviária relativamente ao tamanho de sua população, quando comparado aos demais países europeus. Esses dados podem justificar a eficiência ambiental do país, já que segundo Thompson (2010), o modo ferroviário é mais eficiente ambientalmente do que o modo rodoviário, já que pesquisas comprovaram que se em todo o mundo o tráfego de carga ferroviária fosse transferido para caminhões, a emissão mundial de $\mathrm{CO} 2$ aumentaria em mais de dois por cento.

Já a Lituânia possui uma boa localização geográfica, em que é atravessada por dois corredores de transporte europeus, tendo como resultado um melhor planejamento da malha de transporte. Concomitantemente, possui uma rede ferroviária robusta, em que cerca de $60 \%$ de suas cargas são transportadas via ferrovia. Investimentos em ferrovias ampliariam o número de passageiros transportados, o que tenderia a aumentar a eficiência ambiental do país. Embora as rodovias e as ferrovias necessitem de investimento para melhoria de suas condições, o país conta um alto potencial acadêmico de estudos na área de transporte para superar este ponto a desenvolver (ECORYS Transport, 2006b).

Por fim, com esta análise qualitativa embasada em um aprofundamento da literatura, confirmam-se os resultados quantitativos da ferramenta. Entretanto, ressalta-se que a ferramenta DEA utilizada realiza uma análise pontual dos anos analisados, mas não uma análise prospectiva. Além disso, os dados são referentes à operação e não à construção das ferrovias e rodovias dos países analisados. 


\section{Conclusão}

A partir dos resultados e análises apresentadas, o presente estudo possibilitou uma análise da eficiência ambiental dos modos de transporte rodoviário e ferroviário dos países em questão, mostrando-se relevante tendo em vista a aplicação da modelagem DEA em estudo que relacione as questões de impacto ambiental e emissão de gases poluentes com o setor de transportes.

Em relação à modelagem propriamente dita, destaca-se a utilização do modelo híbrido não decrescente, tendo em vista as especificidades do modelo e as análises geradas pela aplicação do BCC e do CCR previamente. Além disso, ressalta-se também a aplicação do DEA em camadas tendo em vista a queda acentuada das eficiências no ranking do CCR, sinalizando que os países eficientes poderiam estar num nível de eficiência muito elevado, prejudicando assim a ordenação dos demais países.

Ao analisar países que se destacaram através da aplicação do modelo, vale ressaltar que integrantes da União Europeia se sobressaíram na análise de eficiência, o que está alinhado com o fato da União Europeia possuir regras e controles ambientais muito rígidos.

Além disso, outros fatores específicos também podem ser considerados, como o fato de a Letônia apresentar um sistema ferroviário considerado muito forte, em que cerca de $60 \%$ da carga é transportada pelas ferrovias, contribuindo bastante para a redução das emissões de gases poluentes. Já em relação à Lituânia, fatores como a boa localização geográfica, a qualidade de suas rodovias e o bom desenvolvimento das redes existentes contribuem para a eficiência do país analisado.

Entretanto, convém realizar um estudo qualitativo mais aprofundado com o intuito de entender os principais aspectos que contribuem para a eficiência desses países, assim como as estratégias adotadas no setor de transporte que podem ter contribuído para estes resultados.

Outros estudos futuros podem ser sugeridos, como por exemplo, a realização de uma análise com países com características mais homogêneas, principalmente no que diz respeito à extensão territorial e PIB setorial, a fim de ter análises mais específicas. Outro estudo diz 
respeito a uma análise temporal mais completa, com o intuito de identificar possíveis fatores que levaram a eficiência dos países.

Por fim, novos estudos também podem ser feitos utilizando uma abordagem por modo de transporte separadamente ou então com uma análise isolada em relação ao transporte de cargas e de passageiros. Vale mencionar a dificuldade em relação ao acesso a informações confiáveis e oficiais para a elaboração de estudos mais aprofundados.

\section{Referências}

Angulo Meza, L., Biondi Neto, L., Soares de Mello, J.C.C.B., Gomes, E.G., Coelho, P.H.G. Free software for Decision Analysis a software package for Data Envelopment models (2005) ICEIS 2005 - Proceedings of the 7th International Conference on Enterprise Information Systems, pp. 207-212.

Azevedo, G. H. I., Roboredo, M. C., Aizemberg, L., Silveira, J. Q. and Mello, J. C. C. B. S., (2012). Uso de análise envoltória de dados para mensurar eficiência temporal de rodovias federais concessionadas. Journal of Transport Literature, vol. 6, n. 1.

Ballou, H. R. (2010). Gerenciamento da Cadeia de Suprimentos/Logística Empresarial. ARTMED.

Barr, R.S.; Durchholz, M.L., Seiford, L. (2000). Peeling the DEA onion: Layering and rank-ordering DMUs using tiered DEA. Southern Methodist University Technical Report, USA.

Bastos, A. L. D., Baum, D. M., Dias, D. P. (2007). Ruídos e Emissões no Transporte Aéreo. Journal of Transport Literature, vol. 1, n. 1, pp. 66-104.

Gomes, E.G., Soares De Mello, J.C.C.B., Souza, G.D.S., Angulo Meza, L., Mangabeira, J.A.D.C. Efficiency and sustainability assessment for a group of farmers in the Brazilian Amazon (2009) Annals of Operations Research, 169 (1), pp. 167-181.

ECORYS Transport (2006a). Study on Strategic Evaluation on Transport Investment Priorities under Structural and Cohesion funds for the Programming Period 2007-2013. Country Report Latvia. European Commission.

ECORYS Transport (2006b). Study on Strategic Evaluation on Transport Investment Priorities under Structural and Cohesion funds for the Programming Period 2007-2013. Country Report Lithuania. European Commission.

European Comission, 2010, GEIPOT, 2010 apud Andrade, Mattei (2011). Consumo energético e emissões de co2: uma análise do setor de transportes brasileiro. Associação Nacional dos Centros de Pós-Graduação em Economia.

International Transport Forum. Transport Greenhouse Gas Emission - Country Data (2010). Disponível em http://www.internationaltransportforum.org/Pub/pdf/10GHGCountry.pdf. Acesso em 08 de dezembro de 2011.

International Transport Forum. Transport Outlook (2011). Disponível em http://www.internationaltransportforum.org/Pub/pdf/11Outlook.pdf. Acesso em 12 de dezembro de 2011. 
Li, X.-B., Reeves, G.R. Multiple criteria approach to data envelopment analysis (1999) European Journal of Operational Research, 115 (3), pp. 507-517.

Macedo, A. M., Mello, J. C. C. B. S., Gomes, E. G. (2010). Modelo para redistribuição de cotas de emissão de gases do efeito estufa baseado em medidas de eficiência técnica. Gest. Prod., São Carlos, v. 17, n. 3, p. 513-524.

OCDE - Organização para Cooperação e Desenvolvimento Econômico. (2008) Policy instruments to limit negative environmental impacts from increased international transport. Global Forum on Transport and Environment in a Globalising World. México.

OECD - Organização para Cooperação e Desenvolvimento Econômico. (2010) Globalisation, Transport and the Environment, OECD Publishing.

Ramanathan, R. (2005). Estimating Energy Consumption of Transport Modes in India Using DEA and Application to Energy and Environmental Policy. The Journal of the Operational Research Society, Vol. 56, No. 6, pp. 732-737.

Senge-sc - Sindicato dos Engenheiros no Estado de Santa Catarina (2011). Ferrovias: grandes as tentativas. Disponível em: <www.senge-sc.org.br/portal/?center=artigos\&id_artigos=25>. Acesso em: 5 de dez de 2011.

Thompson, L (2010). A vision for railways in 2050. Transport and innovation: unleashing the potential. International Transport Forum.

Tschaffon, P. B. (2011). Um estudo de outputs indesejáveis em DEA com aplicação no setor de distribuição de energia elétrica. Dissertação de mestrado. Universidade Federal Fluminense, Mestrado em Engenharia de Produção. 